\title{
Handling Memory Cache Policy with Integer Points Countings
}

\author{
Philippe Clauss \\ ICPS, Université Louis Pasteur, Strasbourg, \\ Pôle API, Bd Sébastien Brant, \\ 67400 Illkirch France \\ clausseicps.u-strasbg.fr \\ http://icps.u-strasbg.fr
}

\begin{abstract}
We propose an automatic method allowing the computation of useful information on the distribution of data in the memory cache. The number of distinct memory locations touched by a loop is computed. It is shown that these problems are relevant to the same general mathematical problem, which is the counting of the exact number of integer points resulting from linear mappings of polytopes.
\end{abstract}

\section{Introduction}

Nowadays processors are designed with a memory hierarchy organized into several levels, each of which is smaller, faster, and more expensive than the level below. The cache is the memory level between CPU and the main memory. It is usually between $1 \mathrm{~KB}$ to $256 \mathrm{~KB}$, and is divided into lines of $4-128$ bytes. The unit of data transfer between main memory and cache is a line. If the same data in the cache is reused, this is called temporal locality. Since the unit of data in cache is a line, once the line is brought into the cache, any access to the data elements in the line is a cache hit. If data in the same cache line are used, this is called spatial locality. If a data is not found in the cache, a cache miss occurs. The cache miss rate is the ratio of cache misses to cache accesses. A cache hit takes usually one cycle, while a cache miss takes $8-32$ cycles. It is ideal for all memory references to hit in the cache, however, since cache size is much smaller than main memory, once new data needs to be brought in from main memory, some data in the cache has to be replaced.

In this work, we make the following contributions. It is shown how the number of distinct memory locations touched by a loop can be automatically determined. Such issues are very important, for example, in order to determine the minimum cache size avoiding any cache miss to occur, or in order to optimize the shape of tiles for minimal communication when partitioning parallel loops $[1,16]$. Anyway, the knowledge of such information helps the mapping process to cache miss rate optimization [8] and can generally be used as a performance prediction tool for parallel programs.

Our application context is the well-known polytope model, where any iteration or array element is represented as an integer point whose coordinates are its indices values. Hence, we propose a geometrical approach to reach our goals, unlike some other ones as W. Pugh in [15], who proposes the manipulation of 
Presburger formulas for equivalent objectives. These are relevant to the same general mathematical problem, which is the counting of the exact number of integral points resulting from linear mappings of polytopes.

We have introduced in some recent works $[3,4]$ an exact counting method of integer points in polytopes, based on the determination of the Ehrhart polynomials of any parameterized polytopes, where many applications to the analysis and transformations of programs have been shown. An application to probabilistic memory disambiguation has also been presented by Ju, Collard and Oukbir in [9]. Let us recall this method by considering the simple example of a rectangle whose definition depends on two parameters $n$ and $m, P_{n, m}=\left\{(i, j) \in \mathcal{Z}^{2} \mid 0 \leq\right.$ $\left.i \leq \frac{n}{2}, 0 \leq j \leq \frac{m}{2}\right\}$. The objective is to compute an analytic formula $E P(n, m)$, giving the number of integer points in $P_{n, m}$ relatively to $n$ and $m$. Such formulas are called Ehrhart polynomials since they are due to the mathematician Eugène Ehrhart [7]. These polynomials are characterized by periodic coefficients, i.e. arrays of rational values, for which each dimension is associated with a parameter, and where the indices of a selected value are given by the modulos of the parameters relatively to the numbers of possible values. An upper bound of these numbers of possible values is given by the denominator of the polytope, i.e. the lcm of the vertices coordinates denominators. The first step is to compute the parametric coordinates of the vertices with the Loechner/Wilde algorithm [13]. The algorithm answer consists in sets of vertices associated with adjacent $p a-$ rameters validity domains. In our example, the vertices are $(0,0),\left(\frac{n}{2}, 0\right),\left(0, \frac{m}{2}\right)$ and $\left(\frac{n}{2}, \frac{m}{2}\right)$, associated with a single parameters validity domain $\{n \geq 0, m \geq 0\}$. Since the degree of $E P(n, m)$ is equal to the dimension of $P_{n, m}$, it is of the form,

$$
E P(n, m)=\left[\begin{array}{ll}
c_{1} & c_{2} \\
c_{3} & c_{4}
\end{array}\right] n m+\left[\begin{array}{ll}
c_{5} & c_{6} \\
c_{7} & c_{8}
\end{array}\right] n+\left[\begin{array}{ll}
c_{9} & c_{10} \\
c_{11} & c_{12}
\end{array}\right] m+\left[\begin{array}{ll}
c_{13} & c_{14} \\
c_{15} & c_{16}
\end{array}\right]
$$

$E P(n, m)$ can also be defined as being an Ehrhart polynomial of the form $\left[c_{1}, c_{2}\right] n+\left[c_{3}, c_{4}\right]$, where each $c_{k}$ is also an Ehrhart polynomial in $m$ of the same form. Hence, the coefficients of $E P(n, m)$ are computed by resolving systems of linear equations. For example, the coefficients $c_{1}$ and $c_{3}$ occurring when $n \bmod 2=0$ are computed by resolving: $\left\{c_{3}=E P(0, m) ; 2 c_{1}+c_{3}=E P(2, m)\right\}$. To be able to resolve this system, $E P(0, m)$ and $E P(2, m)$ have first to be determined. For example, $E P(0, m)=\left[c_{5}, c_{6}\right] m+\left[c_{7}, c_{8}\right]$ is computed by resolving: $\left\{c_{7}=E P(0,0)=1 ; c_{6}+c_{8}=E P(0,1)=1 ; 2 c_{5}+c_{7}=E P(0,2)=2 ; 3 c_{6}+c_{8}=\right.$ $E P(0,3)=2\}$. The values of $E P(0,0), E P(0,1), E P(0,2)$ and $E P(0,3)$ are determined by using a loop scanning $P_{0,1}, \ldots, P_{0,3}$. Finally, the following result is obtained:

$$
E P(n, m)=\left[\begin{array}{lll}
1 / 4 & 1 / 4 \\
1 / 4 & 1 / 4
\end{array}\right] n m+\left[\begin{array}{ll}
1 / 2 & 1 / 4 \\
1 / 2 & 1 / 4
\end{array}\right] n+\left[\begin{array}{ll}
1 / 2 & 1 / 2 \\
1 / 4 & 1 / 4
\end{array}\right] m+\left[\begin{array}{cc}
1 & 1 / 2 \\
1 / 2 & 1 / 4
\end{array}\right]
$$

which can be easily simplified to:

$$
E P(n, m)=\frac{1}{4} n m+\left[\frac{1}{2}, \frac{1}{4}\right]_{m} n+\left[\frac{1}{2}, \frac{1}{4}\right]_{n} m+\left[\begin{array}{cc}
1 & 1 / 2 \\
1 / 2 & 1 / 4
\end{array}\right]_{n, m}
$$

This parametric formula can be evaluated for any value of $n$ and $m$. For example, the number of integer points in $P_{4500,2001}$ is determined by $E P(4500,2001)=$ $\frac{1}{4} \times 4500 \times 2000+\frac{1}{4} \times 4500+\frac{1}{2} \times 2001+\frac{1}{2}=2252126$. 
All of these described calculations are independent of the size parameters of the considered polytope. Hence, the complexity is $\mathcal{O}(1)$ relatively to these parameters. A more precise complexity analysis yields $\mathcal{O}\left(v(d n)^{m}\right)$, where $v$ denotes the number of parameters validity domains, $d$ denotes the denominator of the polytope, $n$ denotes the polytope dimension (the number of free variables), and $m$ denotes the number of parameters. This complexity can be compared to the one of the Omega test, also used for equivalent objectives [15], which has an upper bound of $2^{2^{2^{\mathcal{O}(n)}}}$. However, the Omega test, implemented in the Omega calculator, is dedicated to the resolution of a large field of problems, as exact data dependence analysis for example [14].

The method can also be used to count integer points in non-parametric polytopes. The way is to introduce an artificial parameter $n$, to compute the corresponding Ehrhart polynomial and then to evaluate this polynomial with the value of $n$ corresponding to the initial problem. Unfortunately, this method does not handle mappings of polytopes. However, we show in this paper that its fullness allows the construction of a general method reaching our current objectives.

\section{Counting in linear mappings of polytopes}

Considering linear references of array elements yields the consideration of linear functions. In the polytope model, array elements are represented geometrically as integer points, whose coordinates are their indices, belonging to a polytope $P$. Hence, any mapping function can be modeled by a general linear mapping function of the form:

$$
\begin{aligned}
P & \longrightarrow M \\
\text { linmap }: X & \longmapsto Y=\left[F_{k}(X)+f_{k}\right]_{1 \leq k \leq q}
\end{aligned}
$$

where $F_{k}$ is a linear integer combination on $X$ and $f_{k}$ is an integer constant. Our objective is to determine the number of different values of $Y$ that are reached through linmap. The difficulty of this problem stems from the fact that generally, the reached values are non-continuous, i.e. do not define a polytope in a regular lattice of integer points.

The following example is also handled by W. Pugh in [15] and by us in [3]. In this last work, a different approach has been proposed, which does not allow the consideration of any linear mapping, but only from $\mathcal{Z}^{n}$ to $\mathcal{Z}^{n-1}$.

Example 1. It consists in the following loop nest:

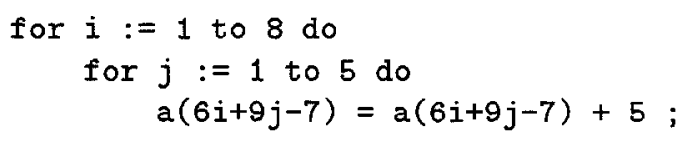

Computing the number of distinct memory locations touched by this loop is equivalent to count the number of distinct values of $6 i+9 j-7$ subject to $1 \leq i \leq 8$ and $1 \leq j \leq 5$. It is also equivalent to count the number of integer points resulting from linmap $(i, j)=6 i+9 j-7$, applied to all the points of the iteration space $P=\left\{(i, j) \in \mathcal{Z}^{2} \mid 1 \leq i \leq 8,1 \leq j \leq 5\right\}$. 
In this example, the mapping is made from dimension 2 to dimension 1. Let $n$ be the dimension of the iteration space and let $q$ be the dimension of the referenced array. The following presented method considers references to any array whose dimension $q$ is greater than one. In cases where $1 \leq q<n$, the problem can be seen as a projection of the iteration space into a lower dimension. In cases where $q>n$, the problem can be seen as an "immersion" in a higher dimensional space. So the objective is to compute the number of integer points resulting from linmap. Hence, this problem is equivalent to determine the number of distinct values of $Y=\left[F_{k}(X)+f_{k}\right]_{1 \leq k \leq q}$ reached through linmap. Let $P_{Y}$ be the parametric polytope defined by:

$$
P_{Y}=\left\{X \in \mathcal{Z}^{n} \mid X \in P, Y=\left[F_{k}(X)+f_{k}\right]_{1 \leq k \leq q}\right\}
$$

Considering any polytope $P_{Y_{0}} \neq \varnothing$, its number of integer points characterizes the number of integer points of $P$ resulting in $Y_{0}$ through linmap. Since our previously recalled counting method allows the computation of the Ehrhart polynomials of the parametric polytope $P_{Y}$, we get expressions of this number of points depending on $Y$ and defined on several adjacent domains $D$ of its values.

Since $P$ is a bounded set of integer points, the set of all the values $Y_{0}$ reached through linmap is also bounded; so is the union of the adjacent domains associated with the Ehrhart polynomials of $P_{Y}$. Unfortunately, these domains also contain values which are not reached through linmap. Hence, the problem is to count exclusively the points of this union which are associated with reached values $Y_{0}$.

Example 2. (continued) The iteration space of the loop nest is modeled by the polytope $P=\left\{(i, j) \in \mathcal{Z}^{2} \mid 1 \leq i \leq 8,1 \leq j \leq 5\right\}$. The parametric polytope characterizing points of $P$ resulting in a given value $y=6 i+9 j-7$, is defined by $P_{y}=\left\{(i, j) \in \mathcal{Z}^{2} \mid 1 \leq i \leq 8,1 \leq j \leq 5, y=6 i+9 j-7\right\}$. Using our method, the Ehrhart polynomials of $P_{y}$ are computed. The answer consists in three adjacent domains $D_{1}, D_{2}$ and $D_{3}$ of the parameter values, each are associated with an Ehrhart polynomial $E P_{1}(y), E P_{2}(y)$ and $E P_{3}(y)$ :

- $D_{1}=\{y \in \mathcal{Z} \mid 8 \leq y \leq 44\}$, $E P_{1}(y)=\left[0,0, \frac{1}{18}\right] y+\left[0,0,-\frac{1}{9}, 0,0,-\frac{5}{18}, 0,0, \frac{5}{9}, 0,0,-\frac{11}{18}, 0,0, \frac{2}{9}, 0,0, \frac{1}{18}\right]$

- $D_{2}=\{y \in \mathcal{Z} \mid 44 \leq y \leq 50\}$, $E P_{2}(y)=[0,0,3,0,0,2]$

- $D_{3}=\{y \in \mathcal{Z} \mid 50 \leq y \leq 86\}$, $E P_{3}(y)=\left[0,0,-\frac{1}{18}\right] y+\left[0,0, \frac{46}{9}, 0,0, \frac{95}{18}, 0,0, \frac{49}{9}, 0,0, \frac{83}{18}, 0,0, \frac{52}{9}, 0,0, \frac{89}{18}\right]$

For example, the array element $a(41)$ is referenced 2 times by the loop: since $41 \bmod 3=2$ (the third value of the first periodic coefficient is selected) and $41 \bmod 18=5$ (the sixth value of the second periodic coefficient is selected), we have $E P_{1}(41)=\frac{41}{18}-\frac{5}{18}=2$.

In the following, some useful information is extracted from the Ehrhart polynomials of $P_{Y}$. Let $E P(Y)$ be an Ehrhart polynomial defined on a domain $D$ of possible reached values $Y$, where the periodic coefficients are expanded to a common size in each given dimension. Let $S$ be the vector of the sizes of any periodic coefficient in all its $q$ dimensions. We denote by $Y \bmod S$ the conjunction 
of all terms $y_{k} \bmod s_{k}, 1 \leq k \leq q$. Let $V=\left(v_{k}\right)$ be a $q$-dimensional vector of integers such that for all $1 \leq k \leq q, 0 \leq v_{k}<s_{k}$. Since an Ehrhart polynomial is characterized by periodic coefficients, it can also be seen as a "periodic polynomial". Hence, for any $Y$ such that $Y \bmod S=V$, a given polynomial occurs:

$$
E P_{V}(Y)=\sum_{k_{1}=0}^{n} \sum_{k_{2}=0}^{n-k_{1}} \ldots \sum_{k_{q}=0}^{n-k_{1}-k_{2}-\ldots-k_{q-1}} c_{k_{1}, k_{2}, \ldots, k_{q}} y_{1}^{i_{1}} y_{2}^{i_{2}} \ldots y_{q}^{i_{q}}
$$

where any $c_{k_{1}, k_{2}, \ldots, k_{q}}$ is a constant rational number.

Proposition 1. If all the coefficients of $E P_{V}(Y)$ are equal to zero, the number of values $Y$ in $D$ reached through linmap and such that $Y \bmod S=V$ is equal to zero. Otherwise, it is equal to $N_{V}-N_{R}$, where $N_{V}$ and $N_{R}$ are defined as follows:

- $N_{V}$ is the number of integer points in the polytope defined by:

$$
\left\{Y \in \mathcal{Z}^{q} \mid Y \in D, Y \bmod S=V\right\}
$$

- $N_{R}$ is the number of integer roots $R$ of $E P_{V}(Y)$ such that $R \bmod S=V$ and $R \in D$.

Proof. It is obvious that if the occurring polynomial $E P_{V}(Y)$, associated with values $Y$ such that $Y \bmod S=V$, has null coefficients, any evaluation of $E P_{V}(Y)$ results in zero. Otherwise, evaluations of $E P_{V}(Y)$ result in zero only for some specific values of $Y$, i.e. the roots of $E P_{V}(Y)$. Hence, if these roots belong to $D$, their values are not reached through linmap and must not be counted. These roots are representative of a phenomenon well-known by the "polytope model" specialists: a polytope projection results in quasi-regular lattices, disrupted by some "bad holes". These prevent to model any projection by its convex hull, making analysis and transformations more difficult. For all other values $Y$ which are not equal to these roots, evaluations of $E P_{V}(Y)$ result in strictly positive integers. Hence, the number of these values is the number of values $Y$ in $D$ reached through linmap and such that $Y \bmod S=V$.

Since the domains $D$ of parameters values are adjacent, they must first be transformed into disjoint ones. This is done easily by replacing some large inequalities by strict inequalities. The following result comes.

Proposition 2. The total number of values reached through linmap is obtained by achieving the previously described counting for all vectors $V$ on each disjoint domain $D$, and by summing all the results.

The last remaining difficulty is to count integer points in the polytopes defined with modulo constraints $Y \bmod S=V$ (proposition 1).

Proposition 3. Let $C$ be a system of constraints defining integer points $Y=$ $\left(y_{k}\right)$, whose non-linear ones are of the form $y_{k} \bmod s_{k}=v_{k}$, where $s_{k}$ and $v_{k}$ are integer constants, $0 \leq v_{k}<s_{k}$.

Let $C^{\prime}$ be the system of linear constraints defining integer points $Z=\left(z_{k}\right)$, deduced from $C$, where any non-linear constraint $y_{k} \bmod s_{k}=v_{k}$ has been deleted and any $y_{k}$, in all the other constraints, has been replaced with $s_{k} z_{k}+v_{k}$.

The number of integer points $Y$ defined by $C$ is equal to the number of integer points $Z$ defined by $C^{\prime}$. 
Proof. Let $y_{0}$ be an integer satisfying $y_{0} \bmod s=v$. This equality implies that there exists an integer $z_{0}$ such that $y_{0}=s z_{0}+v$. Hence, $y_{0}$ can be replaced with $s z_{0}+v$. Making this substitution in a system of constraints allows anticipating the satisfaction of $y \bmod s=v$. The resulting system $C^{\prime}$ defines values $z$ satisfying a system of linear constraints. The values $y$ defined by $C$ can be deduced from $C^{\prime}$ by applying to all $z$ the bijective transformation $s z+v$. Hence, the number of values $z$ satisfying $C^{\prime}$ is equal to the number of values $y$ satisfying $C$.

Example 3. (continued) The domains of the parameters values are transformed into disjoint domains: $D_{1}=\{y \in \mathcal{Z} \mid 8 \leq y \leq 44\}, D_{2}=\{y \in \mathcal{Z} \mid 44<y<50\}$ and $D_{3}=\{y \in \mathcal{Z} \mid 50 \leq y \leq 86\}$. On each domain $D_{p}$ and for every extracted polynomial $E P_{v}(y)$ from $E P_{p}(y)$, such that it is not equal to zero for any value of $y$, we define a polytope whose integer points have to be counted. It yields the following polytopes:

$$
D_{1}\left\{\begin{array} { l } 
{ \{ 8 \leq y \leq 4 4 , y \operatorname { m o d } 1 8 = 2 \} } \\
{ \{ 8 \leq y \leq 4 4 , y \operatorname { m o d } 1 8 = 5 \} } \\
{ \{ 8 \leq y \leq 4 4 , y \operatorname { m o d } 1 8 = 8 \} } \\
{ \{ 8 \leq y \leq 4 4 , y \operatorname { m o d } 1 8 = 1 1 \} } \\
{ \{ 8 \leq y \leq 4 4 , y \operatorname { m o d } 1 8 = 1 4 \} } \\
{ \{ 8 \leq y \leq 4 4 , y \operatorname { m o d } 1 8 = 1 7 \} }
\end{array} D _ { 3 } \left\{\begin{array}{l}
\{50 \leq y \leq 86, y \bmod 18=2\} \\
\{50 \leq y \leq 86, y \bmod 18=5\} \\
D_{2}\left\{\begin{array}{l}
\{44<y<50, y \bmod 18=8\} \\
\{44<y<50, y \bmod 6=86, y \bmod 18=11\} \\
\{50 \leq y \leq 86, y \bmod 18=14\} \\
\{50 \leq y \leq 86, y \bmod 18=17\}
\end{array}\right.
\end{array}\right.\right.
$$

Application of the transformation described in proposition 3 yields the following polytopes:

$$
D_{1}\left\{\begin{array} { l } 
{ \{ 1 \leq z \leq 2 \} } \\
{ \{ 1 \leq z \leq 2 \} } \\
{ \{ 0 \leq z \leq 2 \} } \\
{ \{ 0 \leq z \leq 1 \} } \\
{ \{ 0 \leq z \leq 1 \} } \\
{ \{ 0 \leq z \leq 1 \} }
\end{array} D _ { 2 } \left\{\left\{\begin{array} { l } 
{ \{ 7 < z < 8 \} } \\
{ \{ 7 \leq z \leq 7 \} }
\end{array} D _ { 3 } \left\{\begin{array}{l}
\{3 \leq z \leq 4\} \\
\{3 \leq z \leq 4\} \\
\{3 \leq z \leq 4\} \\
\{3 \leq z \leq 4\} \\
\{2 \leq z \leq 4\} \\
\{2 \leq z \leq 3\}
\end{array}\right.\right.\right.\right.
$$

Since the dimension of the array referenced by the loop is one, counting the number of integer points in these polytopes is obvious. But in the general case, more complicated definitions occur. Hence, counting the number of points in these polytopes yields the use of our counting method on Ehrhart polynomials, applied to non-parametric polytopes, as described in the first section.

In our example, summing all the values $N_{v}$ on each domain $D_{p}$ results in $2+2+3+2+2+2+0+1+2+2+2+2+3+2=27$. For all non-zero polynomials $E P_{v}(y)$, their roots are computed in order to determine $N_{r}$. The computation of the roots of these polynomials can be done using, for example, the function solve provided in Maple. We get the following results: one valid root is found (11), belonging to $D_{1}$. Moreover, one other valid root is found on $D_{3}(83)$. Hence, two has to be subtracted to the first counting determined above. It yields that the number of distinct memory locations touched by the loop nest is equal to 25. This result is the same as the one obtained by W. Pugh in [15].

Example 4. (Application to parametric loop tiling) In order to show the usefulness of such a computation, it is used in the following for parametric loop tiling. Let us modify the loop nest by introducing a size parameter $p$ as follows: 
for $i:=1$ to 8 do

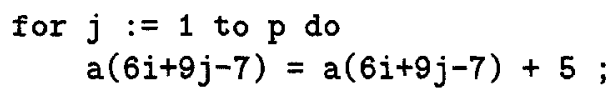

An exact dependence analysis, as described in [5], yields the dependence vector $(3,-2)$. This vector is also called a reuse vector in [11] and [12]. Hence, any iteration $(i+3, j-2)$ uses the array element computed during the iteration $(i, j)$.

A current objective, when mapping such a loop nest on a distributed memory multiprocessor, is to minimize the number of occurring communications. Therefore, mapping on a single processor any iterations $(i, j)$ and $(i+3, j-2)$ yields a communication-free computation of the loop nest and avoids the problem of cache coherence, since the array element $a(6 i+9 j-7)$ is stored in the processor cache. Moreover, this allows to achieve temporal locality.

A current method is to divide the iteration space into tiles, and map them onto the processors. Good shape and size of the tiles allow communication-free blocks and optimized use of the processor cache. As shown in [11], the optimal tile size is difficult to predict. We show in this simple example that our method helps for this prediction.

In our example, communication-free blocks are obtained by choosing a parallelogram shape of the tiles such that one side is generated by the dependence vector $(3,-2)$. Since the upper and lower bounds of index $i$ are constants, the height of the tiles are fixed to the height of the iteration space along the $i$ axis.

Since one tile is mapped onto one processor, array elements accessed by its iterations must fit the cache in order to achieve an optimal use of it. This number of accessed elements is connected with the width of the tiles along the $j$ axis. So the question is, given a cache size of $s$ bytes, what is the width $w$ of the tiles yielding the best use of the cache. Such a parameterized tile yields the computation of a loop on any processor indexed $\left(j_{0} \div(w+1)\right)+1$, such that the innermost loop enumerates all the points of a line segment generated by the dependence vector $(3,-2)$, and the outermost loop enumerates all such lines. This parameterized tile can be defined as a parametric polytope $T_{j_{0}, w}=$ $\left\{(i, j) \in \mathcal{Z}^{2} \mid 1 \leq i \leq 8,1 \leq j_{0}, 3 j_{0}+2 \leq 2 i+3 j \leq 3 j_{0}+3 w+2\right\}$. In order to determine the minimum parametric cache size $s_{w}$ which is necessary to store all the accessed elements, we use our method to compute the number of distinct memory locations touched by the parameterized tile.

By adding the constraint $y=6 i+9 j-7$, we define the parametric polytope $T_{j_{0}, w, y}$ for which the Ehrhart polynomial is computed: $D=\left\{1 \leq j_{0} \leq p-\right.$ $\left.w, 9 j_{0}-1 \leq y \leq 9 j_{0}+9 n-1\right\}, E P\left(j_{0}, w, y\right)=[0,0,2,0,0,3,0,0,3]_{y}$. Then, the number of integer points in $D \cap\{y \bmod 9=2\}, D \cap\{y \bmod 9=5\}$ and $D \cap\{y \bmod 9=8\}$ are computed, yielding $w+w+(w+1)=3 w+1$ distinct memory locations touched. Hence, assuming that any array element is coded on 8 bytes (double float), the minimum cache size allowing an optimal cache utilization, without any occurring cache miss, is $\boldsymbol{s}_{w}=(3 w+1) \times 8$ bytes. For example, a $1 \mathrm{~KB}$ sized cache yields an optimal tiling characterized by $w=42$. 


\section{Conclusion}

While reaching some objectives on cache optimization, the general problem of the number of integer points resulting from any linear mapping of a polytope has been resolved. It has many other applications in the parallelization of nested loops, as the number of allocated processors from linear allocation of iterations, in the same way as in $[2,6]$, or the latency of a parallel loop from a given multidimensional schedule. Non-linear mappings composed of modulos and integer divisions can also be fully considered, by transforming the occurring non-linear constraints into linear ones using the techniques described by W. Pugh in [15]. Other important informations can then be computed, as the number of cache lines touched by a loop, or the number of processors resulting from a distribution (CYCLIC, BLOCK, REPLICATE) of array elements for common languages such as $H P F[10]$.

Since we denote by $e$ the complexity given in section 1 and concerning our exact counting method, the complexity of our counting method in linear mappings is $\mathcal{O}\left(c(v e+1)\right.$ ), where $c$ denotes the number of non-zero polynomials $E P_{V}(Y)$ : the same work is done for all the domains of parameters values, where for each non-zero polynomial, a counting is done using our counting method ; finally, the roots of each of these polynomials are computed.

Ferrante, Sarkar and Thrash in [8] present methods for computing the number of distinct memory locations and cache lines accessed by a loop nest. However, their methods cannot handle unions of polytopes, do not compute symbolic answers, often compute only approximations and use expensive methods to handle the cache lines touched by a set of references. W. Pugh's work in [15], which has been already mentioned, is based on a noticeable different approach, considering a set of constraints as Presburger formulas. The presented technique consists in a set of formulas manipulations, each dedicated to simplify the initial set of constraints. Applying all these tools would be expensive for important sets of constraints. Moreover, their application is not always automatic: it consists in some cases in adding auxiliary variables, and needs the use of standard formulas for sums of powers (the author expects it will be sufficient to hard code the formulas for powers up to 10). This technique also tends to complicated answers when considering rational polytopes. However, the used manipulations of formulas are included in an ambitious package, the Omega calculator, dedicated to the resolution of a large field of problems.

The computation method presented in this paper will be soon added to our implementation on Ehrhart polynomials, in order to complete the objective of an analyzing tool devoted to optimizing parallelization of programs. For future works, we believe that many other computations, which are useful in automatic parallelization, can be achieved through Ehrhart polynomials. Hence, we are currently studying the construction of some other opportune models and methods.

\section{References}

1. A. Agarwal, D.A. Kranz and V. Natarajan. Automatic Partitioning of Parallel Loops and Data Arrays for Distributed Shared-Memory Multiprocessors. IEEE Trans. on Parall. and Distrib. Syst., Vol. 6, NO. 9, Sept. 1995. 
2. Ph. Clauss: An efficient allocation strategy for mapping affine recurrences into space and time optimal regular processor arrays. In C. Jesshope, V. Jossifov and W. Wilhelmi editors, Int. Conf. PARCELLA'94, pages 257-266, Potsdam, Germany, Sept. 1994, Akademie Verlag. http://icps.u-strasbg.fr/pub-94/pub-94-15.ps.z

3. Ph. Clauss: Counting Solutions to Linear and Nonlinear Constraints through Ehrhart polynomials: Applications to Analyze and Transform Scientific Programs. 10th ACM Int. Conf. on Supercomputing, Philadelphia, May 1996. Also available as Research Report ICPS 96-03. http://icps.u-strasbg.fr/pub-96/pub-96-03.ps.gz

4. Ph. Clauss and V. Loechner: Parametric Analysis of Polyhedral Iteration Spaces. IEEE Int. Conf. on Application Specific Array Processors, ASAP'96, Chicago, Illinois, August 1996. Also available as Research Report ICPS 96-04. ht tp://icps.u-strasbg.fr/pub-96/pub-96-04.ps.gz

5. Ph. Clauss and V. Loechner: Parametric Analysis of Polyhedral Iteration Spaces (extended version). To appear in Journal of VLSI Signal Processing, Kluwer Academic Pub., 1997.

6. Ph. Clauss and G.-R. Perrin: Optimal mapping of systolic algorithms by regular instruction shifts. Int. Conf. on Application-Specific Array Processors, ASAP'94, pages 224-235, San Francisco, California, IEEE Computer Society Press. http://icps.u-strasbg.fr/pub-94/pub-94-08.ps.Z

7. E. Ehrhart: Polynômes arithmétiques et Méthode des Polyèdres en Combinatoire. International Serjes of Numerical Mathematics, vol.35, Birkhäuser Verlag, Basel/Stuttgart, 1977.

8. J. Ferrante, V. Sarkar and W. Thrash: On estimating and enhancing cache effectiveness. Advances in Languages and Compilers for Parallel Processing, pages 328-343. The MIT Press, 1991.

9. D. -C. R. Ju, J.-F. Collard and K. Oukbir: Probabilistic Memory Disambiguation and its Application to Data Speculation. Research Report PRiSM 96-010, 1996. ftp://ftp.prism.uvsq.fr/pub/reports/1996/1996.010.ps.gz

10. C. Koelbel, D. Loveman, R. Schreiber, G. Steel, M. Zosel: The High Performance Fortran Handbook. The MIT Press. 1994.

11. M. S. Lam, E. E. Rothberg and M. E. Wolf: The cache performance and optimizations of blocked algorithms. In Proc. 4th Int. Conf. on Architectural Support for Programming Languages and Operating Systems, ASPLOS IV, pages 63-74, Palo Alto, California, April 1991.

12. W. Li: Compiler Optimizations for Cache Locality and Coherence. Technical report 504, University of Rochester, Department of Computer Science, April 1994.

13. V. Loechner and D. K. Wilde: Parameterized polyhedra and their vertices. Research Report ICPS 96-09, 1996.

http://icps.u-strasbg.fr/pub-96/pub-96-09.ps.gz

14. W. Pugh: The Omega test: a fast and practical integer programming algorithm for dependence analysis. Communications of the ACM, 8:102-114, August 1992.

15. W. Pugh: Counting Solutions to Presburger Formulas: How and Why. Proc. of the 1994 ACM SIGPLAN Conference on Programming Language Design and Implementation, 1994.

16. H. S. Stone and D. Thiebaut: Footprints in the cache. Proc. ACM SIGMETRICS 1986, pp. 4-8, May 1986. 